OPEN ACCESS

Edited by:

Saleh A. Naser,

University of Central Florida,

United States

Reviewed by:

Paras Jain,

Albert Einstein College of Medicine,

United States

John T. Belisle,

Colorado State University,

United States

*Correspondence:

Shui-hua Lu

lushuihua66@126.com

Ying Wang

ywang@sibs.ac.cn

${ }^{\dagger}$ These authors have contributed equally to this work.

Received: 12 July 2017 Accepted: 15 November 2017 Published: 30 November 2017

Citation:

Xiao J, Xiong $Y$, Chen $Y$, Xiao $Y$, Ji $P$, Li Y, Wang S, Zhao G, Cheng Q, Lu S and Wang $Y$ (2017) Determination of Lipoprotein Z-Specific IgA in Tuberculosis and Latent Tuberculosis Infection.

Front. Cell. Infect. Microbiol. 7:495. doi: 10.3389/fcimb.2017.00495

\section{Determination of Lipoprotein Z-Specific IgA in Tuberculosis and Latent Tuberculosis Infection}

\author{
Jia-ni Xiao ${ }^{1+}$, Yanqing Xiong ${ }^{2 t}$, Yingying Chen ${ }^{1 \dagger}$, Yang-jiong Xiao ${ }^{1}$, Ping $\mathrm{Ji}^{1}$, Yong $\mathrm{Li}^{1}$, \\ Shu-jun Wang ${ }^{1}$, Guo-ping Zhao ${ }^{3}$, Qi-jian Cheng ${ }^{4}$, Shui-hua Lu ${ }^{2 *}$ and Ying Wang ${ }^{1 *}$ \\ ${ }^{1}$ Department of Microbiology and Immunology, Shanghai Institute of Immunology, Shanghai Jiao Tong University School of \\ Medicine, Shanghai, China, ${ }^{2}$ Key Laboratory of Medical Molecular Virology of MOE/MOH, Shanghai Public Health Clinical \\ Center, Fudan University, Shanghai, China, ${ }^{3}$ Shanghai-MOST Key Laboratory of Health and Disease Genomics, Chinese \\ National Human Genome Center at Shanghai, Shanghai, China, ${ }^{4}$ Department of Respiratory Diseases, Ruijin Hospital, \\ Shanghai Jiao Tong University School of Medicine, Shanghai, China
}

Tuberculosis (TB) remains one of the most severe infectious diseases. It is still of paramount importance to establish more accurate, rapid, and efficient diagnostic methods. Since infection with Mycobacterium tuberculosis $(M . t b)$ is largely mediated through the respiratory tract, IgA responses against mycobacterial proteins are worthy of investigation for their potential clinical utility. In this study, the IgA response targeting lipoprotein Z (LppZ) was determined by using a homemade ELISA with plasma of TB patients $(N=125)$, LTBI individuals ( $N=92)$, healthy controls $(H C s)(N=165)$, as well as TB patients undergoing anti-TB treatment $(N=9)$. In parallel the antigen-specific IFN- $\gamma$ release from PBMCs triggered by LppZ and $M$. th-specific ESAT-6 or CFP-10 was detected by using an ELISPOT assay. It was found that the LppZ-specific IgA level was dramatically higher in TB patients than in HCs $(p<0.0001)$. Compared to that before anti-TB treatment, the LppZ-specific IgA level decreased substantially after 2 months of anti-TB treatment $(\rho=0.0297)$ and remained at low levels until the end of the treatment. What is more, pulmonary TB patients exhibited significantly higher LppZ-specific IgA-values than extra-pulmonary TB patients ( $p=0.0296)$. Interestingly, the LppZ-specific IgA-values were negatively correlated to the amounts of IFN- $\gamma$ released in response to LppZ with statistical significance $(r=-0.5806, p=0.0002)$. LppZ-specific IgA level was also higher in LTBI individuals than in HCs $(p<0.0001)$. Additionally there were some $\mathrm{PPD}^{+} \mathrm{HC}$ individuals with high LppZ-specific IgA levels but the potential of this assay for identifying leaky LTBI in $\mathrm{PPD}^{+} \mathrm{HCs}$ needs to be further investigated through follow-up studies. The sensitivity of detecting TB solely with ESAT-6 or CFP-10-specific IFN- $\gamma$ release was increased by including the LppZ-specific IgA results, respectively, from 86.11 to $100 \%$ and 88.89 to $100 \%$; the sensitivity of screening for LTBI was increased from 80.36 to $83.93 \%$ and 57.14 to $69.64 \%$, respectively. The higher LppZ-specific IgA responses in TB and LTBI populations than in controls indicated high immunoreactivity to LppZ upon $M$. tb infection. Although the assay was not efficient enough for independent application in sero-diagnosis, LppZ-specific IgA might become a complementary biomarker for the improvement of TB and LTBI screening.

Keywords: lipoprotein Z, IgA, tuberculosis, latent tuberculosis infection, screening 


\section{INTRODUCTION}

Tuberculosis (TB), which is caused by Mycobacterium tuberculosis (M. tb) infection, remains one of the biggest infectious disease threats worldwide. One-third of the global population has been infected (Dye et al., 1999). According to the Global Tuberculosis Report 2016 issued by World Health Organization (WHO), there were 10.4 million newly onset TB patients and 1.4 million were killed by TB in 2015. China is among the countries with high TB burden, as demonstrated by the incidence of 918,000 cases and the mortality of 37,600 cases in 2015, ranking third following India and Indonesia (WHO, 2016). Improved TB prevention and control is urgently needed, worldwide and in China, and approaches to optimize current diagnostic methods are of great significance and value.

Currently conventional TB diagnosis mainly relies on clinical symptoms, chest $\mathrm{X}$-rays, and pathogenic microorganism confirmation through sputum microscopy and sputum culture (Baumann et al., 2014). Although identification of mycobacterium bacilli in sputum samples by culture is the gold standard, the long time required together with practical difficulties in bacilli culture limit its successful application in the clinic. The tuberculin skin test (TST) is strongly recommended for TB diagnosis in populations that have not been vaccinated with Bacillus Calmette-Guérin (BCG) (Horsburgh, 2004; Pai et al., 2014). The test is based on a delayed-type hypersensitivity response to purified protein derivative (PPD) containing at least 200 shared antigens of BCG and $M$. $t b$ (Lalvani and Pareek, 2010). Since BCG vaccination is obligatory in China, the TST is not satisfactory either for detecting active TB or for discriminating between $M$. $t b$-infected and uninfected people in a healthy population (Araujo et al., 2008; Zhang et al., 2010). To increase the specificity in TB diagnosis, an $M$. $t b$-specific cellular response-based method, known as an interferon gamma release assay (IGRA) has been developed. This detects IFN- $\gamma$ releasing cells when they are stimulated with two specific antigens, early secretary antigen target-6 (ESAT-6) and culture filtrate protein-10 (CFP-10) (Berthet et al., 1998; Guinn et al., 2004), which are absent from BCG strains and most non-tuberculosis mycobacteria (NTM) (Andersen et al., 2000). Two commercial IGRA kits, QuantiFERON-TB Gold In-Tube (QFT) and T-SPOT.TB, have been applied in TB diagnosis as well as latent tuberculosis infection (LTBI) screening (Ewer et al., 2006; Guio et al., 2011). However, variable sensitivity in detecting active TB in healthy populations and low specificity in distinguishing active TB from LTBI make it necessary to develop alternative diagnostic methods.

Sero-diagnosis is applicable in many infectious diseases such as hepatitis, AIDS etc. However, no successful sero-diagnosis methods have been commercialized for TB based on $M$. $t b$ antigen-specific IgG responses (Baumann et al., 2014; Chen et al., 2015). Owing to the fact that $M$. tb infection is mainly mediated through the respiratory tract, mucosal immunityderived $M$. $t b$-specific IgAs have been demonstrated to play important roles in the defense against M. tb (Kerr, 1990; Casadevall, 2017). IgA-deficient mice were found to be more susceptible to Mycobacterium bovis infection and had higher bacterial loads in the lungs and bronchoalveolar lavage as well as lower IFN- $\gamma$ and TNF- $\alpha$ production in the lungs when compared to wild-type mice (Rodríguez et al., 2005). One recent study revealed that $M$. tb-specific IgA antibody could inhibit mycobacterial infection in an epithelial cell line while IgG antibody promoted $M$. $t b$ infection (Zimmermann et al., 2016). Moreover, purified secretory IgA from human colostrum mediated protection against $M$. $t b$ infection in mice (Alvarez et al., 2013). In addition to the demonstration of protective roles, elevated IgA responses targeting $M$. $t b$ antigens have been evaluated for their potential in diagnosis. For instance, Bezerra et al. (2009) reported that compared to healthy controls (HCs), pulmonary TB (PTB) patients displayed specific IgA response to glycolipid antigen with an assay sensitivity of $88 \%$ and the specificity of $89 \%$. Legesse et al. (2013) described the presence of increased levels of IgA against ESAT-6/CFP-10 and Rv2031 in the sera of culture-confirmed PTB patients when compared to healthy $M$. $t b$-infected and non-infected individuals. IgA targeting other $M$. $t b$ antigens, such as LAM, PE35 (Baumann et al., 2014), P-90 (Conde et al., 2004), PstS3, MPT83 (Baumann et al., 2015) was also detectable in $M$. $t b$-infected individuals.

Mycobacterial lipoproteins have been extensively studied due to their "double-edged sword" features, contributing to both virulence and immunity during $M$. $t b$ infection (Becker and Sander, 2016). Mycobacterial lipoproteins function in nutrient uptake, drug export, cell wall homoeostasis, and hostpathogen interaction (Rezwan et al., 2007; Buddelmeijer, 2015). Lipoprotein Z (LppZ), encoded by $r v 3006$, is a conserved lipoprotein classified in the category of "enzymes and metabolic activities" (Sutcliffe and Harrington, 2004). It has been reported as a novel B cell antigen (Sartain et al., 2006) and one of the most immunogenic proteins with high antibody-to-protein ratio among culture filtrate proteins (Målen et al., 2008). Our previous study revealed that LppZ exhibited strong cellular immunoreactivity in active TB (Xiao et al., 2016). In this study, we determined IgA immune responses to LppZ among three human cohorts: TB patients, LTBI individuals and HCs. Antigenspecific IFN- $\gamma$ release in response to LppZ, ESAT-6, or CFP-10 was detected in parallel. The potential value of LppZ-specific IgA for TB and LTBI screening was also evaluated.

\section{MATERIALS AND METHODS Study Populations}

TB patients $(N=125)$, LTBI individuals $(N=92)$, and HCs $(N=165)$ were included in the study. TB patients were inpatients from Shanghai Public Health Clinical Center (Shanghai, China). TB was confirmed based on medical history, chest radiograph (X-ray and $\mathrm{CT}$ ), acid-fast bacilli (AFB) smear or sputum culture. All the patients were both HIV-negative and HBV-negative. TB patients enrolled in the follow-up study were out-patients undergoing conventional anti-TB chemotherapy from Ruijin Hospital affiliated to Shanghai Jiao Tong University School of Medicine. Thirty-one onset patients were recruited at the starting point of the treatment, among them nine patients fulfilled the criteria of our study with the collection of samples at all-time points. Those who were older than 80 years old $(N=1)$, 
had virus infection $(N=1)$, or other diseases $(N=4)$ including diabetes, thrombocytopenia, etc., were excluded from this study. Sixteen individuals dropped out of the study before the end of the treatment. All TB patients had signed voluntary informed consent before being enrolled in this study.

LTBI individuals and HCs were from healthy blood donors undergoing annual physical examination in Ruijin Hospital (Shanghai, China). All healthy donors had no medical history or disease symptoms. LTBI individuals were defined as those with $\geqq 6$ spot forming units (SFUs) in T-SPOT assay. All individuals involved in this study were adults who had been vaccinated with the BCG Shanghai strain (Shanghai Institute of Biological Products Co., Ltd., Shanghai, China) during childhood. This study was approved by the Ethical Committee of Shanghai Jiao Tong University School of Medicine.

\section{Preparation of $M$. tb Antigens}

The recombinant plasmid pMRLB.54 containing gene rv3006 (Protein LppZ) from M. tb (GeneBank accession No. NR13304) was obtained from NIH Biodefense and Emerging Infection Research Resources Repository (NIAID, NIH, USA). The recombinant-expressing plasmids were transformed to Escherichia coli BL21 (DE3) and LppZ protein was induced for 3$4 \mathrm{~h}$ with $0.5 \mathrm{mM}$ isopropyl $\beta$-D-1-thiogalactopyranoside (IPTG) (Beyotime, Jiangsu, China) at $30^{\circ} \mathrm{C}$. Inducibly expressed protein was purified from bacteria through affinity chromatography with Ni-NTA His-Bind Resin (Qiagen, Duesseldorf, Germany). Lipopolysaccharide (LPS) was removed from purified LppZ protein by using Triton X-114 two-phase separation for at least six cycles (Jensen et al., 2008). In brief, Triton X-114 (Sigma, MO, USA) was added to the purified protein at the final concentration of $1 \%$ and incubated for $30 \mathrm{~min}$ at $4^{\circ} \mathrm{C}$ with constant agitation, followed by incubation for $10 \mathrm{~min}$ at $37^{\circ} \mathrm{C}$ and centrifugation at $16,000 \times g$ for $10 \mathrm{~min}$ at $25^{\circ} \mathrm{C}$. The remaining Triton $\mathrm{X}-114$ was removed by ultrafiltration against PBS. The amount of LPS was measured by Tachyleus Amebocyte Lysate Kit (Gulangyu, Xiamen, China). Those preparations containing LPS lower than 0.10-0.15 EU/mL (which is the FDA endotoxin limit for drugs) were subjected to further experiments. Protein concentration was detected by BCA Protein Assay Kit (Thermo Fisher, MA, USA).

\section{SDS-PAGE and Western Blot}

The purity of recombinant LppZ protein was determined by $12 \%$ sodium dodecyl sulfate polyacrylamide gel electrophoresis (SDSPAGE) (Tanon, Shanghai, China) followed by Coomassie brilliant blue staining and further confirmed by Western blotting using anti- $6 \times$ His tag antibody (Abcam, Hong Kong, China) or human plasma (including TB patient, LTBI individual, HC and disease control). LppZ protein was electro-transferred to polyvinylidene fluoride (PVDF) membrane (Millipore, MA, USA). Human plasma was diluted at 1:200 ratio in the immunostaining assay. Horseradish peroxidase (HRP)-conjugated goat anti-mouse IgG (Cwbio, Beijing, China) and HRP-conjugated goat anti-human IgA (Cwbio) were used as secondary antibodies. The ECL solution (Millipore) was used to visualize LppZ. The membranes were scanned on Tanon 5200S Chemiluminescence Imaging System (Tanon).

\section{Peptide Synthesis}

Nineteen peptides were synthesized by Sangon Biotech (Shanghai, China). These peptides were 20 amino acid residues in length and covered the entire sequences of ESAT- 6 or CFP-10 with overlapping 10-amino-acid residues.

\section{Peripheral Blood Mononuclear Cell Isolation and Plasma Collection}

Whole blood $(10 \mathrm{~mL})$ was collected in tubes containing ethylene diamine tetraacetic acid (EDTA). Peripheral blood mononuclear cells (PBMCs) were isolated by Ficoll-hypaque density gradient centrifugation with Lymphoprep ${ }^{\mathrm{TM}}$ solution (AXIS-SHIELD Poc AS, Oslo, Norway) according to the manufacturer's recommendation. The plasma was collected and stored at $-80^{\circ} \mathrm{C}$. The mononuclear cell layer was carefully transferred to a new $15 \mathrm{~mL}$ conical tube and washed twice with RPMI 1640 medium (GIBCO, NY, USA) by centrifuging at $486 \times g$ for $10 \mathrm{~min}$ at room temperature. $\mathrm{PBMCs}$ were re-suspended at a concentration of $2.5 \times 10^{6} / \mathrm{mL}$ in RPMI 1640 culture medium containing $10 \%$ fetal bovine serum (FBS) (Millipore), 100 units/mL penicillin (GIBCO), and $100 \mu \mathrm{g} / \mathrm{mL}$ streptomycin (GIBCO).

\section{Determination of LppZ-Specific IgA in Plasma}

LppZ-specific IgA levels in human cohorts were determined by homemade enzyme-linked immunosorbent assay (ELISA). Briefly, 96-well polystyrene plates (Corning, NY, USA) were coated with $1 \mu \mathrm{g} / \mathrm{mL}$ purified LppZ protein diluted in bicarbonate/carbonate coating buffer $\left(15 \mathrm{mM} \mathrm{Na}_{2} \mathrm{CO}_{3}\right.$ and $35 \mathrm{mM} \mathrm{NaHCO} 3, \mathrm{pH}$ 9.6) and incubated at $4{ }^{\circ} \mathrm{C}$ overnight. After washing three times with PBST (PBS containing 0.05\% Tween-20; Sangon Biotech), the wells were blocked with 200 $\mu \mathrm{L} /$ well buffer $\mathrm{G}$ (PBS containing $1 \%$ pork gelatin; Sigma) at $37^{\circ} \mathrm{C}$ for $1 \mathrm{~h}$. Human plasma ( $100 \mu \mathrm{L} /$ well, 1:100 diluted in buffer $\mathrm{G}$ ) was added to the wells and incubated at $37^{\circ} \mathrm{C}$ for $1 \mathrm{~h}$. After washing three times with PBST, the wells were incubated with HRP-conjugated goat-anti-human IgA (SouthernBiotech, AL, USA) working solution ( $100 \mu \mathrm{L} /$ well, diluted in buffer $\mathrm{G})$ at $37^{\circ} \mathrm{C}$ for $1 \mathrm{~h}$. Tetramethylbenzidine (TMB) (BD Bioscience, CA, USA) solution $(100 \mu \mathrm{L} /$ well $)$ was added and the plates were incubated at room temperature in the dark for $15 \mathrm{~min}$. The reaction was stopped by adding $1 \mathrm{M} \mathrm{H}_{2} \mathrm{SO}_{4}(50 \mu \mathrm{L} /$ well $)$. The absorbance at $450 \mathrm{~nm}$ was detected within $5 \mathrm{~min}$ by PowerWaveXS2 microplate spectrophotometer (BioTek Instruments, Inc., VT, USA).

\section{Interferon-Gamma (IFN- $\gamma$ ) Release Assay}

Antigen-specific IFN- $\gamma$ releasing level was determined by using enzyme-linked immunospot (ELISPOT) assays according to the manufacturer's instructions (U-CyTech, Utrecht, Netherlands). Briefly, 96-well PVDF plates (Millipore) were coated with anti-human IFN $-\gamma$ coating antibody overnight at $4^{\circ} \mathrm{C}$. The wells were blocked for $1 \mathrm{~h}$ at $37^{\circ} \mathrm{C}$ then $2.5 \times 10^{5}$ PBMCs in $100 \mu \mathrm{L}$ culture medium were plated in each well and stimulated with ESAT-6 or CFP-10 peptide pool $(2 \mu \mathrm{g} / \mathrm{mL}$ per peptide), or $20 \mu \mathrm{g} / \mathrm{mL}$ tuberculin PPD (Statens Serum Institute, 


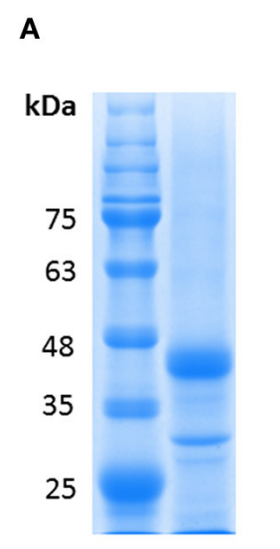

B

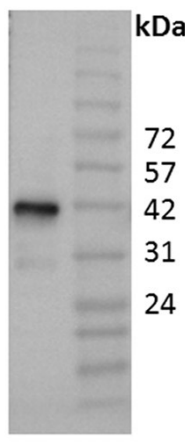

C
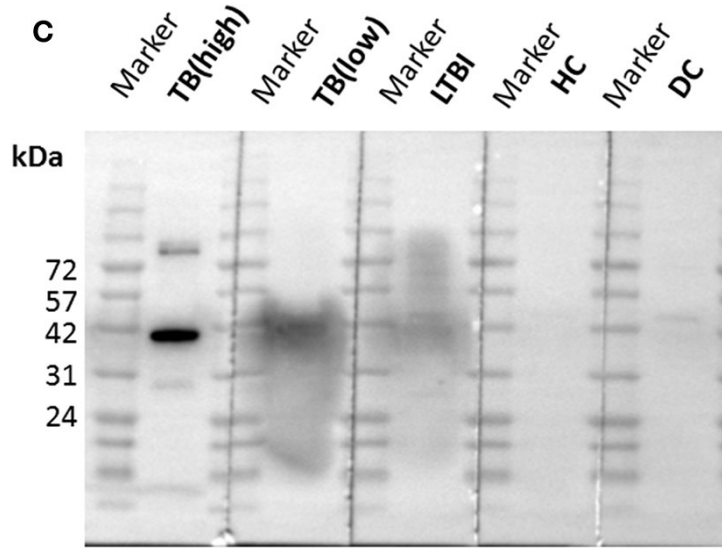

FIGURE 1 | Expression and identification of LppZ protein. (A) SDS-PAGE analysis of purified LppZ protein expressed in E. coli. LppZ was purified by Ni-NTA column and applied to $12 \%$ SDS-PAGE. LppZ protein was visualized through staining with Coomassie brilliant blue. (B) Western blotting of purified LppZ protein. Purified His-tagged LppZ was detected with HRP conjugated anti-6 $\times$ His tag mouse antibody. (C) Western blotting of purified LppZ protein. Purified LppZ was detected with plasma from two TB patients, one LTBI individual, one HC, and one disease control (DC).

in TB and LTBI individuals but minimal in $M . t b$ non-infected individuals.

\section{TB Patients Exhibit Dramatic LppZ-Specific IgA Responsiveness in the Periphery}

To quantitatively determine LppZ-specific IgA levels in the plasma, a homemade ELISA was performed and LppZ-specific IgA levels were compared between TB patients $(N=125)$ and HCs $(N=165)$. The LppZ-specific IgA level was significantly higher in plasma from TB than from HCs $(p<0.0001)$ (Figure 2A) and dramatically higher from PTB patients $(N=63)$ than from EPTB patients $(N=19)(p=0.0296)$ (Figure 2C). In addition, the LppZ-specific IgA level was monitored in the follow-up study over the course of anti-TB treatment, including at the time of diagnosis (month 0 ) and at months 1, 2, 4, and 6. Compared to month 0, LppZ-specific IgA levels had decreased significantly at months 2,4 , and 6 after the treatment $(p=0.0297$, $p=0.0058, p=0.0283$, respectively) (Figure 2D). However, even at month $6 \mathrm{LppZ}$-specific IgA level was still higher than that in HCs. We also determined the AUC-value of LppZ-specific IgA based on a ROC-curve and this reached $0.7657(p<0.0001)$ (Figure 2B).

Thus, the LppZ-specific IgA level was significantly higher in TB patients and more dramatically in PTB patients. With the decline of LppZ-specific IgA levels during anti-TB treatment, there was a presumptive correlation with the bacillary load.

\section{Negative Correlation between LppZ-Specific IgA Level and Cellular Immune Response}

These results indicated that LppZ was an immune dominant antigen capable of inducing strong IgA humoral response. Our previous work indicated that it could also trigger specific cellular immune responses. The relationship between LppZspecific humoral and cellular responses was further analyzed. LppZ-specific release of IFN- $\gamma$ from PBMCs of TB $(N=36)$ and $\mathrm{HC}$ subjects $(N=47)$ was assayed in parallel. Similar to our previous results, the frequency of cells releasing IFN- $\gamma$ in response to LppZ was significantly higher in the TB group than in HCs $(p<0.0001)$ (Figure 3A) and this was accompanied by higher LppZ-specific IgA level in the TB group than that in $\mathrm{HCs}$ in the same cohorts $(p<0.0001)$ (Figure 3B). Analysis of the correlation between LppZ-specific IFN- $\gamma$ SFUs and LppZ-specific IgA-values in the TB group indicated that those patients with higher LppZ-specific IgA levels exhibited lower LppZ-specific IFN- $\gamma$ SFUs, or vice versa (Figure 3C). According to Spearman rank test, there was a significant negative correlation between LppZ-specific IgA levels and LppZ-specific IFN- $\gamma$ releasing levels in TB patients $(r=-0.5806, p=0.0002)$ (Figure 3D).

These data demonstrated that LppZ-specific IgA and IFN$\gamma$ releasing levels were both significantly higher in TB patients than in HCs. However, either the IgA humoral or the IFN- $\gamma$ cellular immune response to LppZ was dominant in individual TB patients.

\section{Determination of LppZ-Specific IgA Levels in LTBI Individuals}

Since LppZ induced strong IgA responses when there was active TB pathogenesis and a detectable $M$. $t b$ bacilli burden, it was questionable whether the response would also be found under latent TB infection (LTBI). When LppZ-specific IgA level was compared between LTBI individuals $(N=92)$ and HCs $(N=165)$, the LppZ-specific IgA level in LTBI individuals was significantly higher than that in HCs $(p<0.0001)$ (Figure 4A). The ROC-curve showed an AUC-value of $0.7075(p<0.0001)$ (Figure 4B). These results further demonstrated that LppZ is a dominant mycobacterial antigen, inducing strong IgA responses even in latent infection by the $M$. $t b$ pathogen. 
A

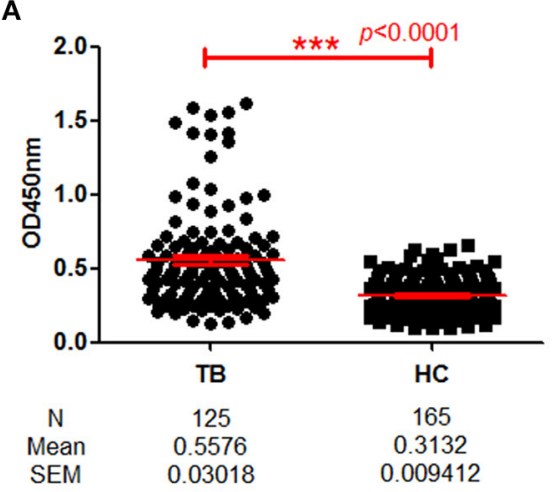

C
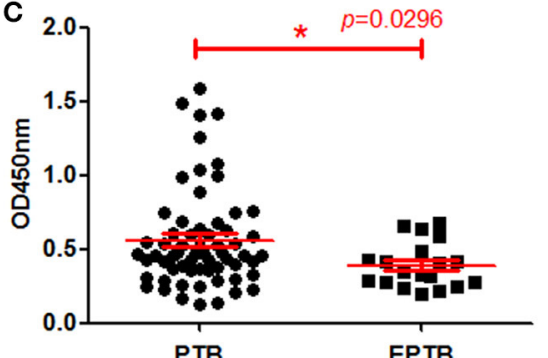

PTB

$\begin{array}{cc}\text { N } & 63 \\ \text { Mean } & 0.5599\end{array}$

SEM 0.04208
EPTB

19

0.3902

0.03527
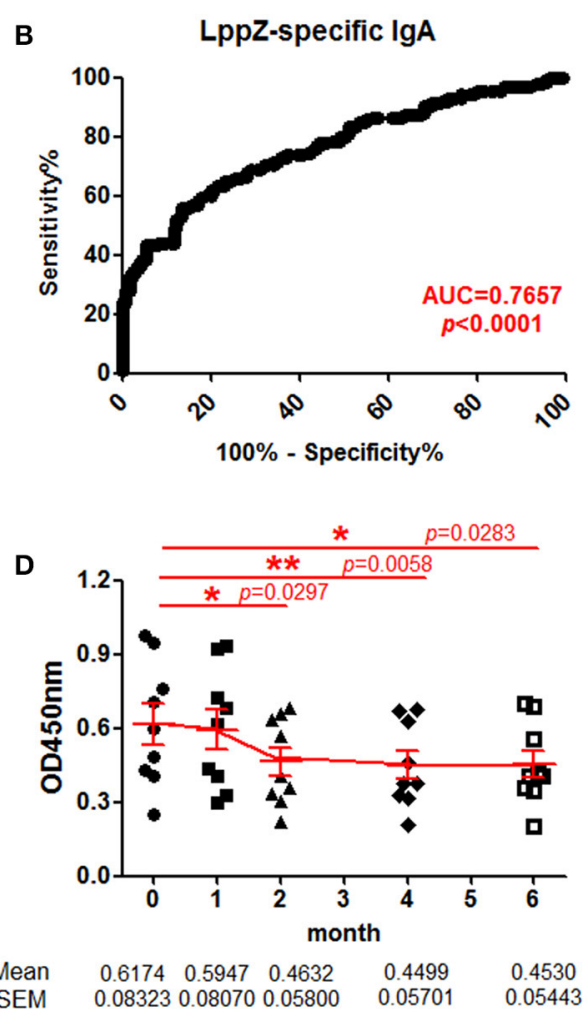

FIGURE 2 | Determination of LppZ-specific IgA level in TB patients. (A) Comparison of optical density $\left(\mathrm{OD}_{450}\right)$-values of LppZ-specific IgA in the plasma between TB patients $(N=125)$ and HCs $(N=165)$. The P-value was calculated using Mann-Whitney test. (B) Receiver operating characteristic (ROC)-curve for LppZ-specific IgA to discriminate TB $(N=125)$ from HCs $(N=165)$. Area under curve (AUC) was 0.7657 (95\% Cl, 0.7096-0.8218). (C) Comparison of OD 450 -values of LppZ-specific IgA in pulmonary TB (PTB) patients $(N=63)$ and extra-pulmonary TB (EPTB) patients $(N=19)$. The P-value was calculated using Mann-Whitney test. (D) Cumulative $\mathrm{OD}_{450}$-values of LppZ-specific IgA in TB patients $(N=9)$ over the course of anti-TB treatment, including the time of diagnosis (month 0 ) and the time points at month $1,2,4,6$ during the treatment. The $P$-value was calculated using paired $t$-test. ${ }^{\star} 0.01<p<0.05,{ }^{\star \star} 0.001<p<0.01,{ }^{\star \star \star} p<0.001$.

\section{Inclusion of the LppZ-Specific IgA Assay Increases the Sensitivities of ESAT-6 and CFP-10-Specific IFN- $\gamma$ Release Assays for}

\section{TB and LTBI Screening}

Since the LppZ-specific IgA level was elevated both in TB patients and LTBI individuals, the potential utility of this finding was investigated. Based on the cutoff value of LppZ-specific IgA in the TB group (0.375), which was determined based on ROCcurve analysis and maximal YI, assay of LppZ-specific IgA alone had a sensitivity of $86.11 \%$ and specificity of $72.12 \%$ in TB patients $(N=36)$ (Table 3$)$. However, when the IgA results were coordinated with ESAT-6 or CFP-10 specific IFN- $\gamma$ releasing levels (Supplementary Figure 2), the diagnostic sensitivities of the ESAT- 6 and CFP-10-specific IFN- $\gamma$ release assays for TB patients were substantially improved from 86.11 to $100 \%$ and 88.89 to $100 \%$, respectively, and the specificities remained at 72.12 and $72.12 \%$ (Table 3 ). In the LTBI cohort (Supplementary Figure 3 ), the cutoff value of LppZ-specific IgA in LTBI was 0.4385 . When this assay was combined with ESAT- 6 or CFP-10-specific IFN- $\gamma$ releasing assays in screening LTBI, the sensitivities were elevated from 80.36 to $83.93 \%$ and from 57.14 to $69.64 \%$, with the specificities of 85.45 and $84.85 \%$, respectively (Table 4 ).
Therefore, although the assay of LppZ-specific IgA is not good enough on its own for clinical application, it can increase the sensitivities of ESAT- 6 or CFP-10-specific IFN- $\gamma$ release assays in TB and LTBI screening, suggesting the potential value of LppZ-specific IgA as an adjunctive screening biomarker.

\section{Individuals with Enhanced LppZ-Specific IgA Levels in the PPD ${ }^{+}$Healthy Group}

Currently, LTBI screening in healthy populations relies mainly on detecting a positive IGRA result in individuals with no clinical symptoms. However, this still cannot exclude the possibility that there exist some undetected leaky LTBI individuals among HCs. In our study, we have performed the IGRA assay in 221 healthy volunteers among whom 56 individuals were identified as LTBI based on SFUs-values $\geqq 6$ in tests with ESAT- 6 or CFP-10. Because PPD has an auxiliary role in TB diagnosis, PPD-specific IFN- $\gamma$ releasing levels were also assayed in LTBI individuals $(N=56)$ and HCs $(N=165)$ in parallel (Figure 5A). Increased PPDspecific IFN- $\gamma$ releasing levels were observed in LTBI individuals (Figure 5B). On ROC-curve analysis the cutoff value of the PPD-specific IFN- $\gamma$ releasing level was calculated as 42.5 SFUs using maximal YI-value (Figure 5C) and this divided HCs into 


\section{A}
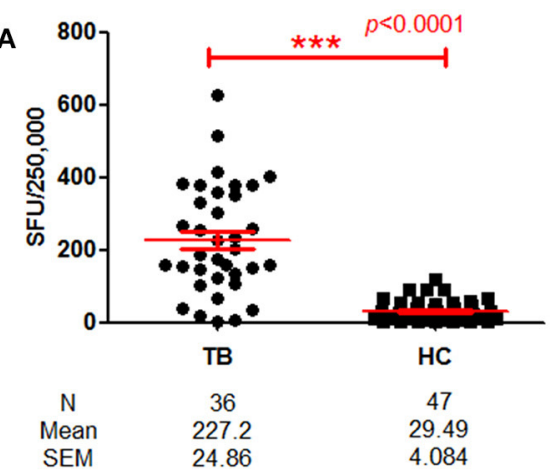

C

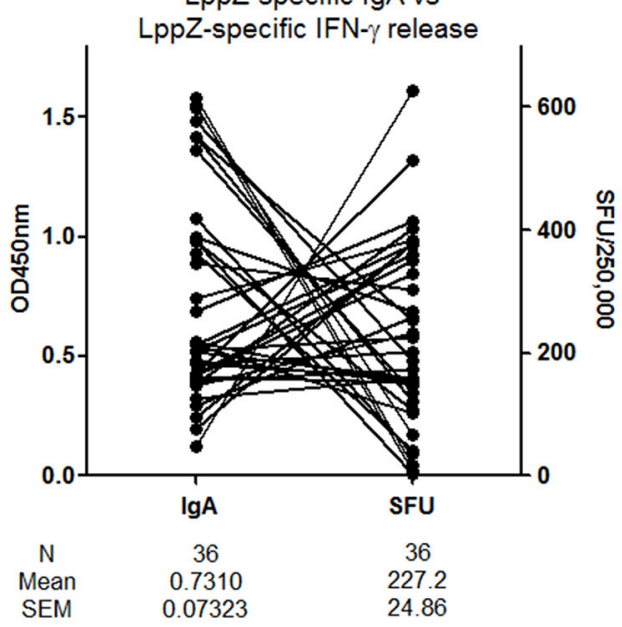

B

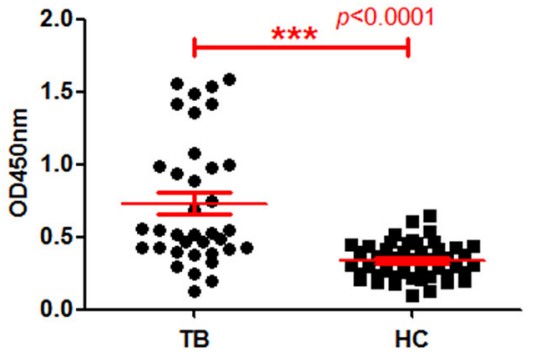

$\begin{array}{ccc}N & 36 & 47 \\ \text { Mean } & 0.7310 & 0.3370 \\ \text { SEM } & 0.07323 & 0.01809\end{array}$

D

LppZ-specific IgA vs LppZ-specific IFN- $\gamma$ release

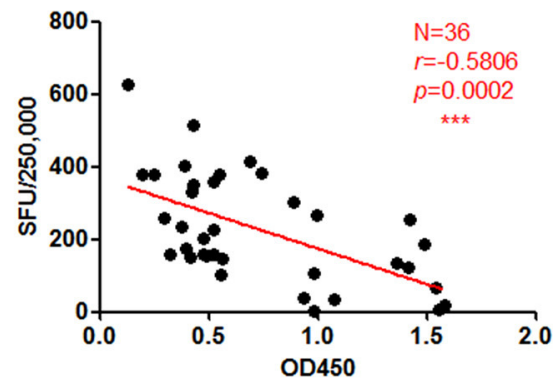

FIGURE 3 | Correlation between LppZ-specific IgA and LppZ-specific IFN- $\gamma$ releasing level in TB patients. (A) LppZ-specific IFN- $\gamma$ releasing cell numbers in PBMCs from TB patients $(N=36)$ and $\mathrm{HCs}(N=47)$. The $P$-value was calculated by using Mann Whitney test. (B) $\mathrm{OD}_{450}$-values of $L p p Z$-specific IgA in TB patients $(N=36)$ and HCs $(N=47)$. The $P$-value was calculated by using Mann Whitney test. (C) Complementarity analysis between LppZ-specific IgA and LppZ-specific IFN- $\gamma$ releasing level in TB patients $(N=36)$. Each line represented one individual. (D) Correlation analysis between LppZ-specific IgA and LppZ-specific IFN- $\gamma$ releasing level in TB patients $(N=36)$. The correlation coefficient $r(r=-0.5806)$ and the $P$-value $(p=0.0002)$ were calculated using the Spearman rank test. SFU: Spot forming unit. ${ }^{\star \star *} p<0.001$
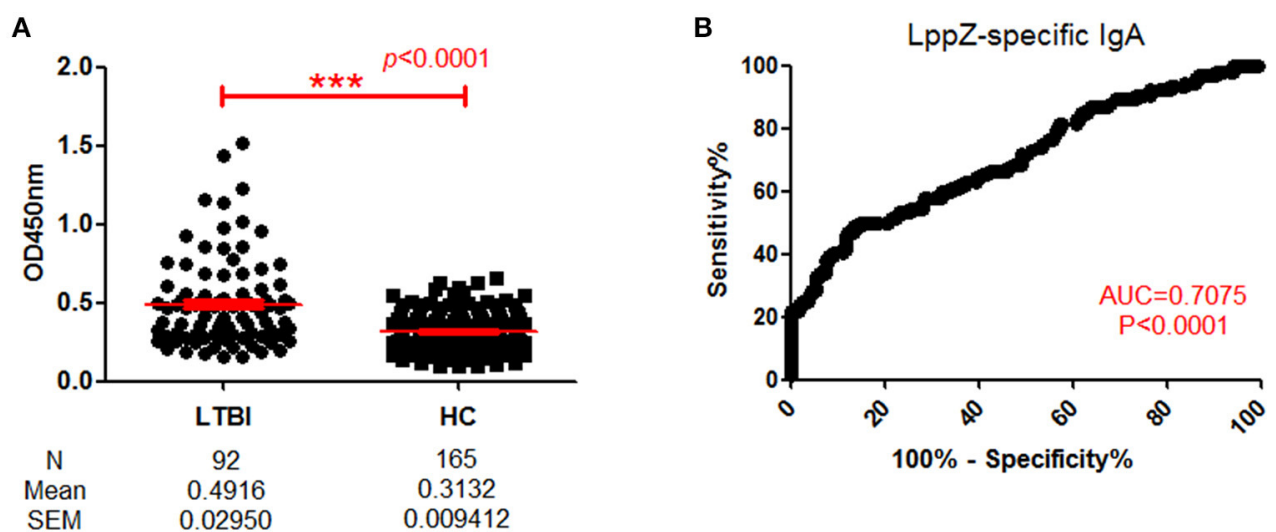

FIGURE 4 | Determination of LppZ-specific IgA level in LTBI individuals. (A) OD 450 -values of LppZ-specific IgA in LTBI ( $N=92)$ and HCs ( $N=165)$. The $P$-value was calculated using Mann Whitney test. (B) ROC-curve for LppZ-specific IgA to discriminate LTBI individuals from HCs. The $P$-value was calculated using Mann Whitney test. AUC was $0.7075(95 \% \mathrm{Cl}, 0.6396-0.7754) .{ }^{\star \star *} p<0.001$ 
TABLE 3 | The sensitivity and specificity of LppZ-specific IgA assay and ESAT-6 or CFP-10-specific IFN- $\gamma$ release assay for TB diagnosis ${ }^{\#}$.

\begin{tabular}{|c|c|c|c|}
\hline & $\begin{array}{l}\text { Cutoff } \\
\text { value }\end{array}$ & $\begin{array}{c}\text { Sensitivity (\%) } \\
\text { (95\% Cl) }\end{array}$ & $\begin{array}{c}\text { Specificity (\%) } \\
(95 \% \mathrm{Cl})\end{array}$ \\
\hline LppZ-specific IgA & 0.375 & $86.11(70.50-95.33)^{\star \star \star}$ & $72.12(64.62-78.81)$ \\
\hline $\begin{array}{l}\text { ESAT-6 specific } \\
\text { IFN- } \gamma \text { releasing }\end{array}$ & 5 & $86.11(70.50-95.33)^{\star \star \star}$ & $100(97.79-100.0)$ \\
\hline $\begin{array}{l}\text { CFP-10 specific } \\
\text { IFN- } \gamma \text { releasing }\end{array}$ & 7 & $88.89(73.94-96.89)^{\star \star \star}$ & 99.39 (96.67-99.98) \\
\hline $\begin{array}{l}\text { LppZ-specific lgA } \\
+ \text { ESAT- } 6 \text { specific } \\
\text { IFN- } \boldsymbol{\gamma} \text { releasing }\end{array}$ & - & $100(-)$ & $72.12(-)$ \\
\hline $\begin{array}{l}\text { LppZ-specific IgA } \\
+ \text { CFP-10-specific } \\
\text { IFN- } \boldsymbol{\gamma} \text { releasing }\end{array}$ & - & $100(-)$ & $72.12(-)$ \\
\hline
\end{tabular}

TABLE 4 | The sensitivity and specificity of LppZ-specific IgA assay and ESAT-6 or CFP-10-specific IFN- $\gamma$ release assay for LTBI screening ${ }^{\#}$.

\begin{tabular}{|c|c|c|c|}
\hline & $\begin{array}{l}\text { Cutoff } \\
\text { value }\end{array}$ & $\begin{array}{l}\text { Sensitivity (\%) } \\
(95 \% \mathrm{Cl})\end{array}$ & $\begin{array}{c}\text { Specificity (\%) } \\
\text { (95\% Cl) }\end{array}$ \\
\hline LppZ-specific lgA & 0.4385 & $32.14(20.29-45.96)^{\star \star \star}$ & 85.45 (79.13-90.45) \\
\hline $\begin{array}{l}\text { ESAT-6 specific } \\
\text { IFN- } \boldsymbol{\gamma} \text { releasing }\end{array}$ & 5 & $80.36(67.57-89.77)^{\star \star \star}$ & $100(97.79-100.0)$ \\
\hline $\begin{array}{l}\text { CFP- } 10 \text { specific } \\
\text { IFN- } \gamma \text { releasing }\end{array}$ & 5 & $57.14(43.22-70.29)^{\star \star \star}$ & 99.39 (96.67-99.98) \\
\hline $\begin{array}{l}\text { LppZ-specific IgA } \\
+ \text { ESAT- } 6 \text { specific } \\
\text { IFN- } \gamma \text { releasing }\end{array}$ & - & $83.93(-)$ & $85.45(-)$ \\
\hline $\begin{array}{l}\text { LppZ-specific IgA } \\
+ \text { CFP-10-specific } \\
\text { IFN- } \boldsymbol{r} \text { releasing }\end{array}$ & - & $69.64(-)$ & $84.85(-)$ \\
\hline
\end{tabular}

$\operatorname{PPD}^{+}(N=72)$ and $\operatorname{PPD}^{-}(N=93)$ groups. As shown in Figure 5D, the $\mathrm{PPD}^{+} \mathrm{HC}$ group was further classified into two groups: a $\mathrm{PPD}^{+} \mathrm{E} 6 \mathrm{C} 10^{\text {hi }} \mathrm{HC}$ group $(N=26)$, defined as $\mathrm{PPD}^{+}$HCs with ESAT-6 or CFP-10-specific IFN- $\gamma$ releasing cell numbers $\geqq 3$ and a $\mathrm{PPD}^{+} \mathrm{E} 6 \mathrm{C} 10^{\text {lo }} \mathrm{HC}$ group $(N=46)$, defined as having ESAT-6 and CFP-10-specific IFN- $\gamma$ releasing cell number $\leqq 2$. The LppZ-specific IgA level in the $\mathrm{PPD}^{+} \mathrm{E} 6 \mathrm{C} 10^{\mathrm{hi}}$ $\mathrm{HCs}$ was significantly higher than that in the $\mathrm{PPD}^{+} \mathrm{E} 6 \mathrm{C} 10^{\text {lo }}$ HCs $(p=0.0031)$ (Figure 5E). What is more, there existed 5 $\mathrm{PPD}^{+} \mathrm{E} 6 \mathrm{C} 10^{\mathrm{hi}}$ individuals (highlighted as " $\Delta$ " in Figure $5 \mathrm{E}$ ) who had LppZ-specific IgA levels that were not only higher than the cutoff value $(0.4585)$ but also higher than all $\mathrm{PPD}^{+} \mathrm{E} 6 \mathrm{C} 10^{\text {lo }}$ $\mathrm{HC}$ individuals. Considering that the assay of LppZ-specific IgA level remarkably increased the sensitivity of ESAT-6 or CFP10-specific IGRA assay in LTBI screening, it can be speculated that these $5 \mathrm{PPD}^{+} \mathrm{E} 6 \mathrm{C} 10^{\mathrm{hi}} \mathrm{HCs}$ might be high-risk LTBI candidates.

\section{DISCUSSION}

Nowadays, lipoproteins in $M . t b$ are being increasingly studied in respect to roles in both virulence and immunity (Rezwan et al., 2007; Buddelmeijer, 2015). They display strong immunogenicity that is probably related to their prevalent distribution and abundant expression in mycobacterial pathogens. So far, some of the lipoproteins have been identified as potential biomarker proteins for TB discrimination. For instance, PstS1 (Rv0934), also known as $38 \mathrm{kDa}$ protein, shows great immunogenicity (Målen et al., 2008) with the ability to induce B and T cell responses upon $M$. $t b$ infection (Harboe and Wiker, 1992). Moreover, $38 \mathrm{kDa}$ protein has been recommended to be used in TB sero-diagnosis (Senol et al., 2009). LppZ, as investigated here, also exhibits strong immunogenicity in both humoral and cellular aspects and in both TB and LTBI population. More interestingly, elevated anti-LppZ IgA responses are more evident than IgG responses in TB and LTBI (data not shown). This is probably due to the fact that $M$. $t b$ infection occurs mainly through the respiratory tract. Results from the comparison between PTB and EPTB cases, where $M . t b$ is considered to have less involvement of the respiratory tract in EPTB, also support the conclusion that $\mathrm{TB}$ pathogenesis triggers strong mucosal immunity leading to the induction of secretory IgA. In addition, the substantial decrease in LppZ-specific IgA levels in the follow-up TB patients accompanies the amelioration of the disease at month 2 after anti-TB treatment. Since most of the patients become bacilli negative at month 2 when they finish the intensive phase of anti-TB treatment, our results strongly suggest that the generation of IgA-mediated mucosal immunity is largely due to $M$. $t b$ infection. Similar results are observed in $\operatorname{IgA}$ reactivity to glycolipid antigen after 2 and 6 months during anti-TB therapy, suggesting a potential role for antigenspecific IgA in monitoring pulmonary TB treatment (Bezerra et al., 2009). However, we did not see strong correlation between LppZ-specific IgA levels and disease severity in PTB patients, as indicated by smear positivity, culture positivity and cavitation (Supplementary Figure 4).

Our findings demonstrate that LppZ is of high immunogenicity upon $M . t b$ infection and several studies indicate that both humoral and cellular immune responses are involved in protection against $M$. $t b$ infection (Falla et al., 1991; Mattos et al., 2010; Scriba et al., 2017). The observation that high levels of $M$. $t b$-specific antibodies could be detected without TST reactivity suggests that $M$. $t b$-specific antibodies could be elevated in the absence of cellular immune response (Bothamley et al., 1992; Sousa et al., 1997). In our study, LppZ exhibits both higher cellular and higher IgA responses in TB patients when compared to HCs. Moreover, LppZ-specific IgA levels are negatively correlated to LppZ-specific IFN- $\gamma$ releasing levels in TB patients. We therefore speculate that there might exist a dominant immunity pattern targeting LppZ antigen that differs between individuals upon $M$. $t b$ infection; either humoral or cellular may be selected for dominance.

Because of the elevated LppZ-specific IgA in TB and LTBI cohorts, we intend to evaluate its potential in clinical application. The AUC-values of LppZ-specific IgA (0.7657 for TB diagnosis 
A

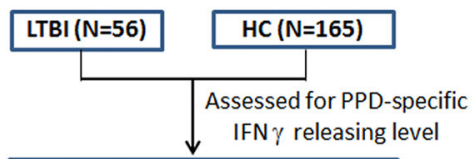

Cutoff value of PPD-specific IFN $\gamma$ releasing level $=\mathbf{4 2 . 5} \mathrm{SFU}$

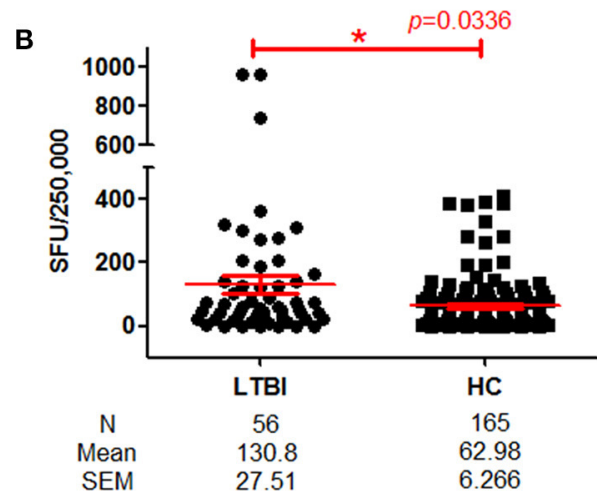

C PPD-specific IFNY releasing cell numbers (LTBIVSHC)

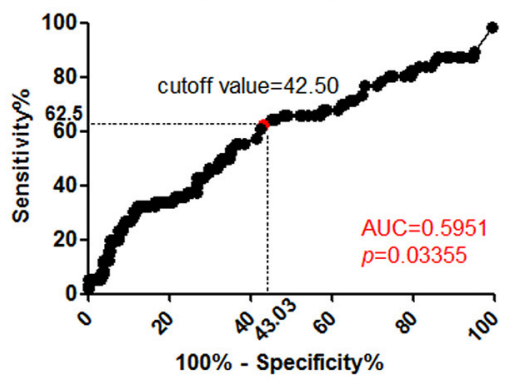

D

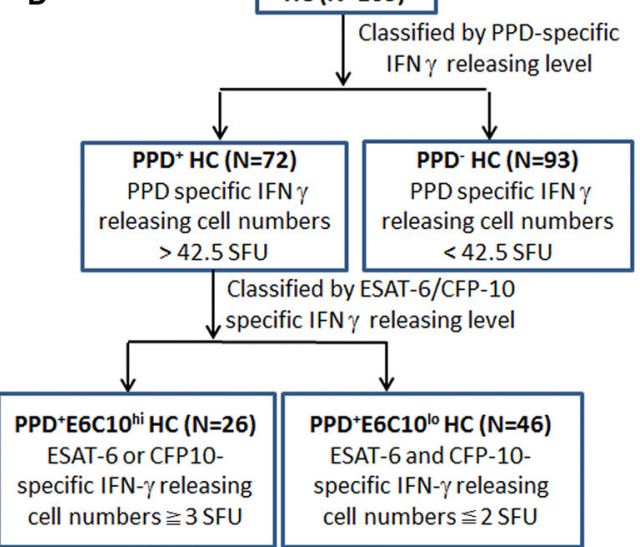

E

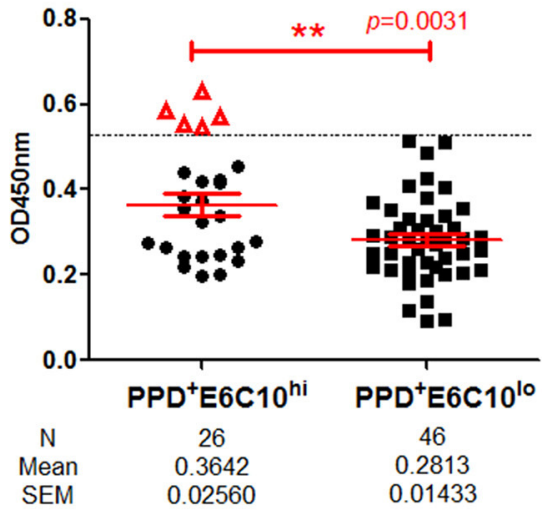

FIGURE 5 | LppZ-specific IgA level in PPD + HC groups. (A) Flow diagram to define the cutoff value of PPD-specific IFN- $\gamma$ releasing levels in HCs. (B) PPD-specific IFN- $\gamma$ releasing cell numbers in LTBI individuals $(N=56)$ and HCs $(N=165)$. The $P$-value was calculated using Mann Whitney test. (C) ROC-curve for PPD-specific IFN- $\gamma$ releasing cell numbers between $L T B I$ individuals $(N=56)$ and HCs $(N=165)$. The cutoff value was calculated using maximal Youden Index. (D) Flow diagram to define PPD ${ }^{+}$E6C10 $10^{\text {hi }}$ and PPD ${ }^{+}$E6C10 lo HCs. (E) LppZ-specific lgA levels of PPD ${ }^{+} E 6 C 10^{\text {hi }} \mathrm{HCs}(N=26)$ and PPD ${ }^{+}$E6C10 ${ }^{\text {lo }} \mathrm{HCs}(\mathrm{N}=46)$. The $P$-value was calculated using Mann Whitney test. $\Delta$ represents those with higher LppZ-specific IgA level than cutoff value (0.4585). PPD ${ }^{+}$HCs: HC individuals with PPD-specific IFN- $\gamma$ releasing cell numbers $>$ cutoff value $(42.5 \mathrm{SFU})$; PPD + E6C10 hi HCs: PPD + HCs with ESAT-6 or CFP-10-specific IFN- $\gamma$ releasing cell numbers $\geqq 3$ SFU; PPD ${ }^{+}$E6C10 ${ }^{l o}$ HCs: PPD ${ }^{+}$HCs with ESAT-6 and CFP-10-specific IFN- $\gamma$ releasing cell numbers $\leqq 2$ SFU. ${ }^{\star} 0.01<p<0.05,{ }^{\star *} p<0.01$.

and 0.7075 for LTBI screening) theoretically imply its potential in discriminating $M$. $t b$-infected individuals from non-infected individuals. However, the LppZ-specific IgA assay has sensitivity and specificity that are too low to fulfill the criteria for either TB diagnosis or LTBI screening when compared with other available diagnostic methods (Tables 3, 4). Thus, the IgA-based test on its own seems unlikely to provide an adequate biomarker for TB and LTBI identification. Consequently we can consider what clinical application there might be for using such strong LppZ-specific IgA responses in TB in the future. At present, M. $t b$-specific ESAT- 6 or CFP-10-based IGRA assays are widely used in distinguishing $M . t b$-infected from $M$. $t b$ non-infected individuals (Pai et al., 2014). However, the specificity and sensitivity are still under question due to the different stages and situations of $M$. $t b$ infection which arise. Recently, Li et al. (2017) demonstrated that higher sensitivity for TB diagnosis could be achieved when combining M. $t b$ antigen Rv3615c with ESAT-6 and CFP-10 as stimulus. Another strategy for improvement is to add extra biomarkers in the assay. Here we show that the combination of measurement of LppZ-specific IgA together with assays of ESAT- 6 or CFP-10 specific IFN- $\gamma$ releasing levels could markedly raise the sensitivity in screening $M$. $t b$-infected individuals (Tables 3, 4). Thus, measurement of LppZ-specific IgA holds significant potential as an adjunctive biomarker for monitoring $M$. $t b$ infection.

It has been well-acknowledged that both TST and IGRA assays can be used to determine the presence of $M$. $t b$-specific T cell responses (Young et al., 2009; Li et al., 2017). While the PPDbased TST does reveal the antigen-specific immune responses to previous infection (Morrison et al., 2008; Young et al., 2009), the IGRA assay reflects $M . t b$ infection more specifically (Hill et al., 2007). For decades, LTBI individuals have been identified mainly on the basis of positive results in either TST or IGRA assays, despite the fact that both methods are deficient to some 
degree in sensitivity. For instance, a proportion of repeatedly TBexposed healthy people never convert into positivity in TST assay (Joshi et al., 2006). ESAT-6/CFP-10 specific cellular responses mainly stand out for their extremely high specificity (usually over 90\%) in diagnosing M. $t b$ infection (van Pinxteren et al., 2000; Harada et al., 2004). By following the protocol of the assay kit manufacturer, subjects with $\geqq 6$ SFUs against synthesized ESAT6 or CFP-10 peptide pools are screened out as LTBI individuals. This standard for screening LTBI individuals has been suspected to be not as sensitive as expected (Hill et al., 2007), indicating the existence of false-negative LTBI individuals during screening. In our study, we sub-grouped HCs based on PPD and ESAT-6/CFP10 specific cellular responses. We speculated that subjects with high IFN- $\gamma$ releasing levels in response to both PPD and ESAT6/CFP-10 were more likely to have $M$. $t b$ infection. Consistent with this, when LppZ-specific IgA level was compared between $\mathrm{PPD}^{+} \mathrm{E} 6 \mathrm{C} 10^{\text {hi }}$ (more than three SFUs upon E6C10 stimulation) and $\mathrm{PPD}^{+}$E6C10 ${ }^{\text {lo }}$ ( $<3$ SFUs) HC subjects, it was shown that not only were the average levels of LppZ-specific IgA higher, but also there existed $5 \mathrm{HCs}$ with higher LppZ-specific IgA levels in the $\mathrm{PPD}^{+} \mathrm{E} 6 \mathrm{C} 10^{\text {hi }}$ group (Figure 5E). The values of LppZ-specific $\mathrm{IgA}$ of five $\mathrm{HCs}$ were higher than those in $\mathrm{PPD}^{+} \mathrm{E} 6 \mathrm{C} 10^{\text {lo }}$ group. Considering the role of LppZ-specific IgA in indicating $M$. $t b$ infection, we presume that those five individuals might be "leaky LTBI" individuals. Whether the assay of LppZ-specific IgA could be applied to increase the sensitivity of LTBI screening needs to be further investigated using cohort studies.

\section{CONCLUSIONS}

In the present study, we report higher LppZ-specific IgA levels in both TB and LTBI groups when compared to HCs, indicating that LppZ is of high immunogenicity upon $M$. $t b$ infection. What's more, combining assay of LppZ-specific IgA with that of ESAT-6 or CFP-10 specific IFN- $\gamma$ releasing levels dramatically increases the sensitivities of TB and LTBI screening when

\section{REFERENCES}

Alvarez, N., Otero, O., Camacho, F., Borrero, R., Tirado, Y., Puig, A., et al. (2013). Passive administration of purified secretory IgA from human colostrum induces protection against Mycobacterium tuberculosis in a murine model of progressive pulmonary infection. BMC Immunol. 14(Suppl. 1), S3. doi: 10.1186/1471-2172-14-S1-S3

Andersen, P., Munk, M. E., Pollock, J. M., and Doherty, T. M. (2000). Specific immune-based diagnosis of tuberculosis. Lancet 356, 1099-1104. doi: 10.1016/S0140-6736(00)02742-2

Araujo, Z., de Waard, J. H., de Larrea, C. F., Borges, R., and Convit, J. (2008). The effect of Bacille Calmette-Guerin vaccine on tuberculin reactivity in indigenous children from communities with high prevalence of tuberculosis. Vaccine 26, 5575-5581. doi: 10.1016/j.vaccine.2008. 08.006

Baumann, R., Kaempfer, S., Chegou, N. N., Oehlmann, W., Loxton, A. G., Kaufmann, S. H., et al. (2014). Serologic diagnosis of tuberculosis by combining Ig classes against selected mycobacterial targets. J. Infect. 69, 581-589. doi: 10.1016/j.jinf.2014.05.014

Baumann, R., Kaempfer, S., Chegou, N. N., Oehlmann, W., Spallek, R., Loxton, A. G., et al. (2015). A subgroup of latently Mycobacterium tuberculosis infected individuals is characterized by consistently elevated IgA compared to using ESAT-6 or CFP-10 specific IFN- $\gamma$ releasing assay alone. Although sero-diagnosis is not recommended by WHO among standard diagnostic methods for TB (WHO, 2010), the high levels of LppZ-specific IgA in both TB and LTBI reveal a potential role in increasing the sensitivity of the present diagnostic methods. It may serve as a complementary biomarker for monitoring disease treatment and $M$. $t b$ infection. Its potential in identifying leaky LTBI in $\mathrm{PPD}^{+}$healthy populations needs to be further investigated through follow-up studies.

\section{AUTHOR CONTRIBUTIONS}

YW and SL designed the experiments. JX, YC, YX conducted the experiments. YW, SL, GZ, JX, and YC analyzed the data. YX, QC, and SL collected the samples and clinical data. PJ, SW, Y-jX, and YL contributed reagents, materials, analysis tools. YW, SL, and JX wrote the manuscript.

\section{ACKNOWLEDGMENTS}

This work was supported by Grants from Chinese National Mega Science and Technology Program on Infectious Diseases (2017ZX10301301-001-004 and 2013ZX10003007-003-003), the National Science Foundation of China (81501361) and the Fundamental Research Funds for the Central Universities. We appreciate Dr. Hao Shen from University of Pennsylvania for advice on project design and data interpretation. We thank Dr. Douglas Lowrie from Tuberculosis Research Network Center for revising the draft of the manuscript.

\section{SUPPLEMENTARY MATERIAL}

The Supplementary Material for this article can be found online at: https://www.frontiersin.org/articles/10.3389/fcimb. 2017.00495/full\#supplementary-material responses to several mycobacterial antigens. Mediators Inflamm. 2015:364758. doi: 10.1155/2015/364758

Becker, K., and Sander, P. (2016). Mycobacterium tuberculosis lipoproteins in virulence and immunity - fighting with a double-edged sword. FEBS Lett. 590, 3800-3819. doi: 10.1002/1873-3468.12273

Berthet, F. X., Rasmussen, P. B., Rosenkrands, I., Andersen, P., and Gicquel, B. (1998). A Mycobacterium tuberculosis operon encoding ESAT-6 and a novel low-molecular-mass culture filtrate protein (CFP-10). Microbiology 144(Pt. 11), 3195-3203. doi: 10.1099/00221287-144-11-3195

Bezerra, J. M., Beck, S. T., Kanunfre, K. A., Leite, O. M., and Ferreira, A. W. (2009). A study of IgA antibody response to different Mycobacterium tuberculosis antigens in the diagnosis and monitoring of pulmonary tuberculosis. Braz. J. Infect. Dis. 13, 53-58. doi: 10.1590/S1413-86702009000100012

Bothamley, G. H., Beck, J. S., Potts, R. C., Grange, J. M., Kardjito, T., and Ivanyi, J. (1992). Specificity of antibodies and tuberculin response after occupational exposure to tuberculosis. J. Infect. Dis. 166, 182-186. doi: 10.1093/infdis/166.1.182

Buddelmeijer, N. (2015). The molecular mechanism of bacterial lipoprotein modification-how, when and why? FEMS Microbiol. Rev. 39, 246-261. doi: 10.1093/femsre/fuu006

Casadevall, A. (2017). Antibodies to Mycobacterium tuberculosis. N. Engl. J. Med. 376, 283-285. doi: 10.1056/NEJMcibr1613268 
Chen, T., Lin, J., Wang, W., Fleming, J., Chen, L., Wang, Y., et al. (2015). Cytokine and antibody based diagnostic algorithms for sputum culture-positive pulmonary tuberculosis. PLOS ONE 10:e0144705. doi: 10.1371/journal.pone.0144705

Conde, M. B., Suffys, P., Lapa, E., Silva, J. R., Kritski, A. L., Dorman, S. E., et al. (2004). Immunoglobulin A (IgA) and IgG immune responses against P-90 antigen for diagnosis of pulmonary tuberculosis and screening for Mycobacterium tuberculosis infection. Clin. Diagn. Lab. Immunol. 11, 94-97. doi: 10.1128/CDLI.11.1.94-97.2004

Dye, C., Scheele, S., Dolin, P., Pathania, V., and Raviglione, M. C. (1999). Consensus statement. Global burden of tuberculosis: estimated incidence, prevalence, and mortality by country. WHO Global Surveillance and Monitoring Project. JAMA 282, 677-686. doi: 10.1001/jama.282.7.677

Ewer, K., Millington, K. A., Deeks, J. J., Alvarez, L., Bryant, G., and Lalvani, A. (2006). Dynamic antigen-specific T-cell responses after point-source exposure to Mycobacterium tuberculosis. Am. J. Respir. Crit. Care Med. 174, 831-839. doi: $10.1164 / \mathrm{rccm} .200511-1783 \mathrm{OC}$

Falla, J. C., Parra, C. A., Mendoza, M., Franco, L. C., Guzman, F., Forero, J., et al. (1991). Identification of B- and T-cell epitopes within the MTP40 protein of Mycobacterium tuberculosis and their correlation with the disease course. Infect. Immun. 59, 2265-2273.

Guinn, K. M., Hickey, M. J., Mathur, S. K., Zakel, K. L., Grotzke, J. E., Lewinsohn, D. M., et al. (2004). Individual RD1-region genes are required for export of ESAT-6/CFP-10 and for virulence of Mycobacterium tuberculosis. Mol. Microbiol. 51, 359-370. doi: 10.1046/j.1365-2958.2003.03844.x

Guio, H., Vilaplana, C., and Cardona, P. J. (2011). Immunodiagnosis and biomarkers in tuberculosis. Med. Clin. 137, 408-413. doi: 10.1016/j.medcli.2010.09.008

Harada, N., Higuchi, K., Sekiya, Y., Rothel, J., Kitoh, T., and Mori, T. (2004). [Basic characteristics of a novel diagnostic method (QuantiFERON TB-2G) for latent tuberculosis infection with the use of Mycobacterium tuberculosis-specific antigens, ESAT-6 and CFP-10]. Kekkaku 79, 725-735.

Harboe, M., and Wiker, H. G. (1992). The 38-kDa protein of Mycobacterium tuberculosis: a review. J. Infect. Dis. 166, 874-884. doi: 10.1093/infdis/166.4.874

Hill, P. C., Brookes, R. H., Fox, A., Jackson-Sillah, D., Jeffries, D. J., Lugos, M. D., et al. (2007). Longitudinal assessment of an ELISPOT test for Mycobacterium tuberculosis infection. PLoS Med. 4:e192. doi: 10.1371/journal.pmed.0040192

Horsburgh, C. R., Jr. (2004). Priorities for the treatment of latent tuberculosis infection in the United States. N. Engl. J. Med. 350, 2060-2067. doi: 10.1056/NEJMsa031667

Huang, F., Zhang, H., Lv, Q., Sato, K. D., Qu, Y., Huan, S., et al. (2016). Use of anti-tuberculosis drugs among newly diagnosed pulmonary tuberculosis inpatients in China: a retrospective study. Infect. Dis. Poverty 5, 2. doi: 10.1186/s40249-016-0098-9

Jensen, L. B., Torp, A. M., Andersen, S. B., Skov, P. S., Poulsen, L. K., Knol, E. F., et al. (2008). The biological activity of a recombinantly expressed (His)-tagged peanut allergen ( $\mathrm{rAra} \mathrm{h} 1$ ) is unaffected by endotoxin removal. J. Immunol. Methods 335, 116-120. doi: 10.1016/j.jim.2008.02.012

Joshi, R., Reingold, A. L., Menzies, D., and Pai, M. (2006). Tuberculosis among health-care workers in low- and middle-income countries: a systematic review. PLoS Med. 3:e494. doi: 10.1371/journal.pmed.0030494

Kerr, M. A. (1990). The structure and function of human IgA. Biochem. J. 271, 285-296. doi: 10.1042/bj2710285

Lalvani, A., and Pareek, M. (2010). A 100 year update on diagnosis of tuberculosis infection. Br. Med. Bull. 93, 69-84. doi: 10.1093/bmb/ldp039

Legesse, M., Ameni, G., Medhin, G., Mamo, G., Franken, K. L., Ottenhoff, T. H., et al. (2013). IgA response to ESAT-6/CFP-10 and Rv2031 antigens varies in patients with culture-confirmed pulmonary tuberculosis, healthy Mycobacterium tuberculosis-infected and non-infected individuals in a tuberculosis endemic setting, Ethiopia. Scand. J. Immunol. 78, 266-274. doi: $10.1111 /$ sji. 12080

Li, G., Li, F., Zhao, H. M., Wen, H. L., Li, H. C., Li, C. L., et al. (2017). Evaluation of a New IFN- $\gamma$ release assay for rapid diagnosis of active tuberculosis in a high-incidence setting. Front. Cell. Infect. Microbiol. 7:117. doi: 10.3389/fcimb.2017.00117

Målen, H., Softeland, T., and Wiker, H. G. (2008). Antigen analysis of Mycobacterium tuberculosis $\mathrm{H} 37 \mathrm{Rv}$ culture filtrate proteins. Scand. J. Immunol. 67, 245-252. doi: 10.1111/j.1365-3083.2007.02064.x

Mattos, A. M., Almeida Cde, S., Franken, K. L., Alves, C. C., Abramo, C., de Souza, M. A., et al. (2010). Increased IgG1, IFN-gamma, TNF-alpha and IL-6 responses to Mycobacterium tuberculosis antigens in patients with tuberculosis are lower after chemotherapy. Int. Immunol. 22, 775-782. doi: 10.1093/intimm/dxq429

Morrison, J., Pai, M., and Hopewell, P. C. (2008). Tuberculosis and latent tuberculosis infection in close contacts of people with pulmonary tuberculosis in low-income and middle-income countries: a systematic review and metaanalysis. Lancet Infect. Dis. 8, 359-368. doi: 10.1016/S1473-3099(08)70071-9

Pai, M., Denkinger, C. M., Kik, S. V., Rangaka, M. X., Zwerling, A., Oxlade, O., et al. (2014). Gamma interferon release assays for detection of Mycobacterium tuberculosis infection. Clin. Microbiol. Rev. 27, 3-20. doi: 10.1128/CMR.00034-13

Rezwan, M., Grau, T., Tschumi, A., and Sander, P. (2007). Lipoprotein synthesis in mycobacteria. Microbiology 153(Pt 3), 652-658. doi: $10.1099 /$ mic. $0.2006 / 000216-0$

Rodríguez, A., Tjarnlund, A., Ivanji, J., Singh, M., Garcia, I., Williams, A., et al. (2005). Role of IgA in the defense against respiratory infections IgA deficient mice exhibited increased susceptibility to intranasal infection with Mycobacterium bovis BCG. Vaccine 23, 2565-2572. doi: 10.1016/.j.vaccine.2004.11.032

Sartain, M. J., Slayden, R. A., Singh, K. K., Laal, S., and Belisle, J. T. (2006). Disease state differentiation and identification of tuberculosis biomarkers via native antigen array profiling. Mol. Cell. Proteomics 5, 2102-2113. doi: 10.1074/mcp.M600089-MCP200

Scriba, T. J., Coussens, A. K., and Fletcher, H. A. (2017). Human immunology of tuberculosis. Microbiol. Spectr. 5. doi: 10.1128/microbiolspec.TBTB2-0016-2016

Senol, G., Ecevit, C., and Ozturk, A. (2009). Humoral immune response against 38- and $16-\mathrm{kDa}$ mycobacterial antigens in childhood tuberculosis. Pediatr. Pulmonol. 44, 839-844. doi: 10.1002/ppul.20901

Sousa, A. O., Salem, J. I., Lee, F. K., Vercosa, M. C., Cruaud, P., Bloom, B. R., et al. (1997). An epidemic of tuberculosis with a high rate of tuberculin anergy among a population previously unexposed to tuberculosis, the Yanomami Indians of the Brazilian Amazon. Proc. Natl. Acad. Sci. U.S.A. 94, 13227-13232. doi: $10.1073 /$ pnas.94.24.13227

Sutcliffe, I. C., and Harrington, D. J. (2004). Lipoproteins of Mycobacterium tuberculosis: an abundant and functionally diverse class of cell envelope components. FEMS Microbiol. Rev. 28, 645-659. doi: 10.1016/j.femsre.2004.06.002

van Pinxteren, L. A., Ravn, P., Agger, E. M., Pollock, J., and Andersen, P. (2000). Diagnosis of tuberculosis based on the two specific antigens ESAT- 6 and CFP10. Clin. Diagn. Lab. Immunol. 7, 155-160. doi: 10.1128/CDLI.7.2.155-160.2000

WHO. (2010). Commercial Serodiagnostic Tests for Diagnosis of Tuberculosis Expert Group Meeting Report.

WHO (2015). Latent TB Infection Factsheet.

WHO (2016). Global Tuberculosis Report.

Xiao, Y., Sha, W., Tian, Z., Chen, Y., Ji, P., Sun, Q., et al. (2016). Adenylate kinase: a novel antigen for immunodiagnosis and subunit vaccine against tuberculosis. J. Mol. Med. 94, 823-834. doi: 10.1007/s00109-016-1392-5

Young, D. B., Gideon, H. P., and Wilkinson, R. J. (2009). Eliminating latent tuberculosis. Trends Microbiol. 17, 183-188. doi: 10.1016/j.tim.2009.02.005

Zhang, S., Shao, L., Mo, L., Chen, J., Wang, F., Meng, C., et al. (2010). Evaluation of gamma interferon release assays using Mycobacterium tuberculosis antigens for diagnosis of latent and active tuberculosis in Mycobacterium bovis BCG-vaccinated populations. Clin. Vaccine Immunol. 17, 1985-1990. doi: 10.1128/CVI.00294-10

Zimmermann, N., Thormann, V., Hu, B., Kohler, A. B., Imai-Matsushima, A., Locht, C., et al. (2016). Human isotype-dependent inhibitory antibody responses against Mycobacterium tuberculosis. EMBO Mol. Med. 8, 1325-1339. doi: $10.15252 / \mathrm{emmm} .201606330$

Conflict of Interest Statement: The authors declare that the research was conducted in the absence of any commercial or financial relationships that could be construed as a potential conflict of interest.

Copyright $\odot 2017$ Xiao, Xiong, Chen, Xiao, Ji, Li, Wang, Zhao, Cheng, Lu and Wang. This is an open-access article distributed under the terms of the Creative Commons Attribution License (CC BY). The use, distribution or reproduction in other forums is permitted, provided the original author(s) or licensor are credited and that the original publication in this journal is cited, in accordance with accepted academic practice. No use, distribution or reproduction is permitted which does not comply with these terms. 